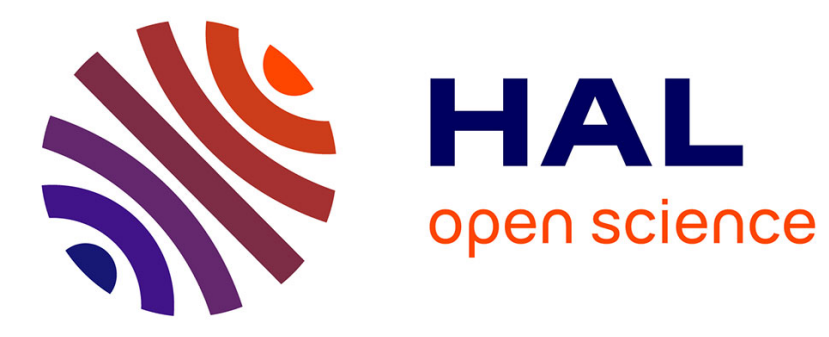

\title{
Implementation and Evaluation of a Satisfaction/Altruism Based Architecture for Multi-Robot Systems
}

Philippe Lucidarme, Olivier Simonin, Alain Liégeois

\section{To cite this version:}

Philippe Lucidarme, Olivier Simonin, Alain Liégeois. Implementation and Evaluation of a Satisfaction/Altruism Based Architecture for Multi-Robot Systems. ICRA: International Conference on Robotics and Automation, May 2005, Washington, United States. pp.1007-1012, 10.1109/ROBOT.2002.1013487 . lirmm-00269416

\section{HAL Id: lirmm-00269416 https://hal-lirmm.ccsd.cnrs.fr/lirmm-00269416}

Submitted on 3 Apr 2008

HAL is a multi-disciplinary open access archive for the deposit and dissemination of scientific research documents, whether they are published or not. The documents may come from teaching and research institutions in France or abroad, or from public or private research centers.
L'archive ouverte pluridisciplinaire HAL, est destinée au dépôt et à la diffusion de documents scientifiques de niveau recherche, publiés ou non, émanant des établissements d'enseignement et de recherche français ou étrangers, des laboratoires publics ou privés. 


\title{
Implementation and Evaluation of a Satisfaction/Altruism Based Architecture for Multi-Robot Systems
}

\author{
P. Lucidarme, O. Simonin, A. Liégeois \\ LIRMM \\ University Montpellier II and CNRS, 161 rue Ada, 34392 Montpellier, France \\ \{lucidarm,simonin,liegeois\}@ lirmm.fr
}

\begin{abstract}
We have developed an agent's architecture towards the goal of building efficient, robust and safe multi-robot systems considered as cooperating distributed reactive agents. This architecture is based on satisfaction and altruism allowing the agents to amend their low-level behavior like goal seeking and collision avoidance in order to solve more complex problems. We demonstrate in particular that local conflicting and locking situations are automatically avoided or made repulsive. Computer simulations of tasks in complex environments confirm it. The designed mini-robots, the implementation of their architecture, and the communication protocol are described. The same hardware is shared between communication, collision avoidance, and task achievement. Experiments using two mobile robots and a test bed confirm the theoretical and simulation results.
\end{abstract}

\section{Introduction}

There are many possible applications for the use of multiple cooperating robotic vehicles and mobile manipulators. The greatest advantage of such teams relies on the distributed sensing, computing and actions. That allows using simple agents which add their capabilities for achieving complex tasks, while ensuring flexibility and adaptation in case of environmental unexpected changes, and self-organization in case of serious failures.

These advantages over a single robot implementation make the autonomous multi-robot systems attractive mostly for applications in hostile and/or remote environments:

- Planet exploration

- Underwater applications using AUVs

- Maintenance and dismantling in nuclear plants

- Defense.

We do not consider here sophisticated agents which are able in particular:
- To compute a map of the universe.

- To compute safe routes, plan the coordinated motions of the various robots, and to re-plan in case of failure.

Such systems [1][2][3], known as "cognitive agents", need precise sensing and large computing facilities, and are sensitive to failures and rapid environmental changes.

In contrast to this approach, this paper is devoted to the type of so-called "reactive" agents, which react immediately to the sensed information thanks to lowresolution sensors and to the limited number of the possible elementary actions.

It has been observed experimentally and by simulations [4] that groups of simple reactive robotic vehicles show many interesting properties:

- Automatic emergence of efficient collective behaviors

- Unsupervised learning and adaptation

Furthermore, reactive robots are cheaper than cognitive ones, and easier to program and to maintain.

Section 2 presents our concept of cooperative-reactive architecture [5], following some general principles already present in the studies of R. Brooks [6], R. Arkin [7] or L. Parker [8] for example, among the extensive research on the subject. The concept is based on a potential field method [9] combining attractive and repulsive forces due to goals, obstacles and signals of other agents in the neighborhood. By this way, the feedback from the sensors to the actuators is practically immediate.

Section 3 describes typical experiments using minirobots. First we present our design of modular minirobots implementing the proposed architecture. Then, the communication protocol used for sharing simple information about each agent's state is presented. Finally, the task and experimental conditions are described.

The detailed analysis and discussion of the experimental results (Section 4) demonstrate that the concept is efficient and can be implemented to solve the conflicts

Finally, it is concluded that our work can be extended to larger teams, heterogeneous ones, and other generic collective tasks. 


\section{The architecture}

Collective systems have great capability of autoorganization and adaptation. However, when implementing this approach on real autonomous robots, it appears that a large number of robots are needed to obtain good performance, which can also result in conflicts [10].

To solve these problems, we have introduced a form of reactive intentional cooperation between "situated" agents. Our method relies on intentional signals exchanged between agents and transformed into move vectors called "altruistic" reactions.

The architecture uses two concepts of agent's satisfaction. First, the personal satisfaction (noted P) measures the agent's progress in the task. It is a signed value that is continuously updated from internal and external perceptions.

Secondly, the interactive satisfaction I evaluates the interaction between the agent and its neighbors. It can either be positive, negative or neutral. If negative, e.g. in case of hindrance or conflict, the agent emits a repulsive signal: a negative I value. By contrast, if the agent needs help or wants to share an abundant resource, an attractive signal I (a positive value) is emitted. An example of frustration situation (hindrance) is illustrated in Figure 1.

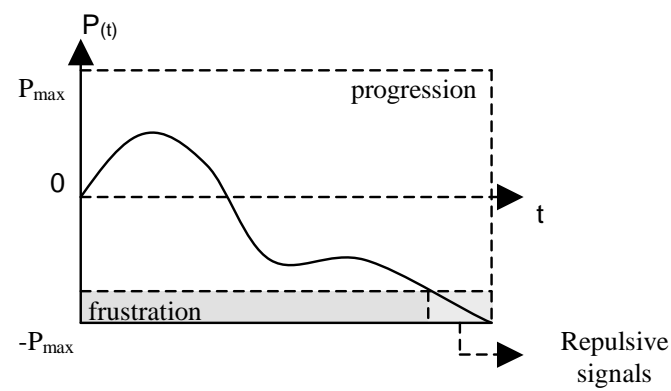

Figure 1: Personal satisfaction

When an agent perceives a signal it can have an altruistic reaction by stopping its current task and moving to satisfy the request. If multiple signals are received, the agent moves according to the signal having the maximal absolute value $\left|\mathrm{I}_{\mathrm{m}}\right|$.

An agent decides to be altruist or not by comparing its personal satisfaction $(\mathrm{P})$ and the signal intensity $\left|\mathrm{I}_{\mathrm{m}}\right|$. If the agent chooses to satisfy the request it moves by applying the altruism vector deriving from a signed potential field. For an agent $\mathrm{B}$ that receives a signal $\mathrm{I}_{\mathrm{A}}$ of an agent $\mathrm{A}$, the altruism vector is computed as

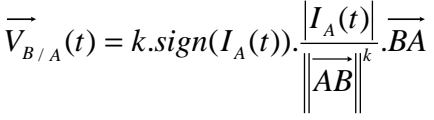

Thus, the goal of the agent may become this vector, combined with obstacle avoidance vectors. The principle of the agent's architecture is given in Figure 2.

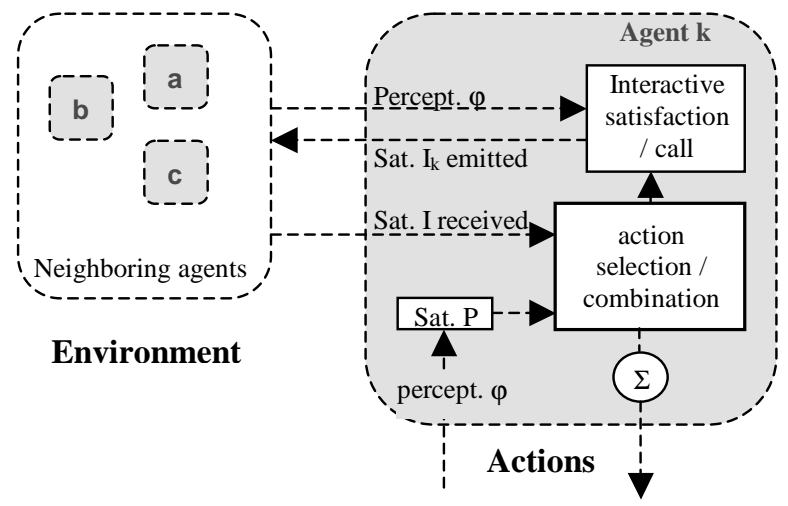

Figure 2: The altruistic agent architecture.

The ability of the model to improve the performance of a reactive system on foraging and collective navigation tasks has been previously demonstrated [5][11].

Moreover, the possibility to transmit messages has been added. Thus, an initial attractive signal may be diffused between agents in order to efficiently recruit many agents [5].

The same principle exists for repulsive signals but it is implicit. When an agent perceives a repulsive signal and if it cannot move to be altruist because of another, it will be dissatisfied and send a repulsive signal. This implicit propagation is useful to escape deadlock situations as shown in Figure 3. This ability has been developed and implemented on real robots and is presented in this paper.

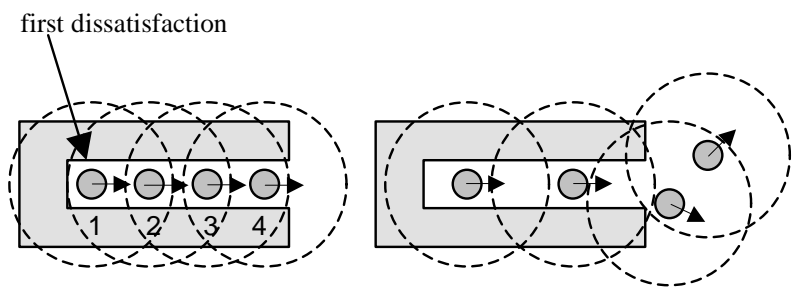

Figure 3: Principle of repulsive signal passing

To ensure that the agents perform "intelligent" signal passing, two rules have been integrated:

Rule 1: when an agent perceives a signal of dissatisfaction $\mathrm{Ie}<0$ stronger than its own $(|\mathrm{Ie}|>|\mathrm{I}|)$ it becomes altruist and passes a signal equal to $\mathrm{Ie}+\varepsilon$ ( $\varepsilon$ being the resolution of the signal's value).

Rule 2: when an agent perceives a signal of dissatisfaction Ie $<0$ lower than its own $(|\mathrm{Ie}|<|\mathrm{I}|)$, it does not use it and its signal $\mathrm{I}$ is equal to its personal dissatisfaction $\mathrm{P}<0$. 
The first rule ensures the propagation of dissatisfaction of the most constrained agents. In signal passing, the $\varepsilon$ variation creates a gradient of intensity, which represents the direction of the diffusion. It is useful in case of disconnection during communication.

When an agent does not perceive a more dissatisfied one than itself, the second rule defines the signal of dissatisfaction as the personal dissatisfaction of the agent.

From these two simple rules, the agents can prevent spatial conflicts and solve them. We present in the following sections the implementation and experimentation of these local rules and the resulting efficient behaviors.

\section{The experiments}

To complement our study, the proposed architecture has been tested on a real problem. As the architecture is based on real-time navigation combining goal attraction, obstacle avoidance and reaction to simple signals, it can manage several generic tasks such as foraging, consuming and collective navigation. This paper focuses on the problem of multi-robot navigation in very constrained environments. Before introducing this application, let us describe the robots on which the satisfaction/altruism model has been implemented.

\subsection{Hardware}

To complete any task of foraging, grazing or consuming, each robot must be able to perform the following elementary behaviors:

- Move

- Communicate at a local level

- Detect obstacles

- Process local information in real time.

Our prototype is shown on Figure 4.a. The robot is a $10 \mathrm{~cm}$ high and $13 \mathrm{~cm}$ diameter cylinder. The robot is composed of several levels similar to those of the previous version [12]. Each electronic board is on top of the other. The main processor is an embedded PC and the boards communicate via the PC 104 Bus.

The first level is the actuated chassis. It has a cylindrical shape and is based on two differential wheels shown on Figure 4.b. The motor can drive the robot at the maximum speed of $1 \mathrm{~m} . \mathrm{s}^{-1}$. Motors are equipped with encoders. Each part of the chassis has been milled and lightened. Batteries are placed on the chassis and not shown on the pictures. The two Ni-MH batteries provide more than half an hour of autonomy.

The second level is the first electronic board. This board drives the motors, supplies the embedded PC and controls the motors and the encoders.

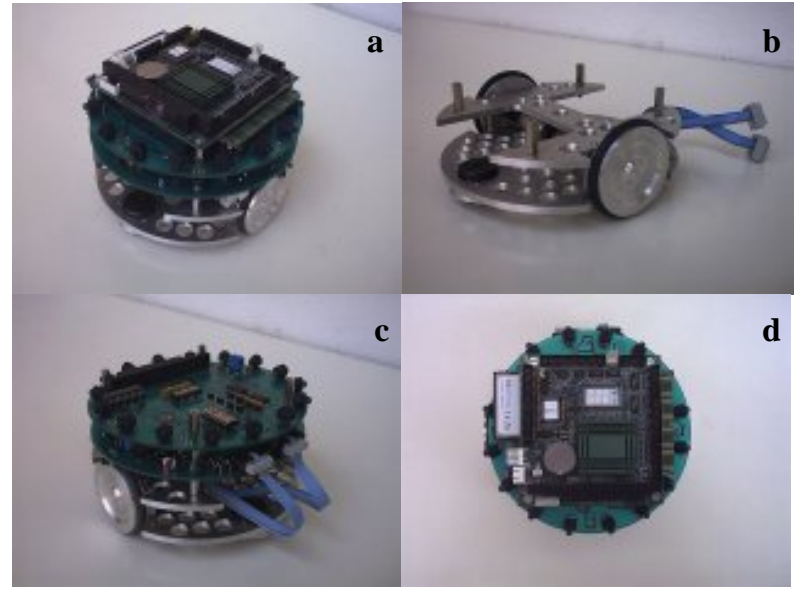

Figure 4: Prototype of an agent

The second electronic board is the infrared board. The robot uses the same sensors to communicate with other robots and to avoid obstacles. Thanks to these sensors, the robot can differentiate other agents from obstacles. The board is surrounded with 16 infrared emitters and 8 receivers (Figure 4.c). The sensors use a carrier frequency of $40 \mathrm{kHz}$ ensuring a good noise rejection.

The last board, called the motherboard, is presently an embedded PC. The processor is an 80486 DX with a 66 $\mathrm{MHz}$ clock. This board is on the top of the robot, which enables the user to add other modules on the robot. Figure 4.d shows a top view of the motherboard.

\subsection{A communication protocol for situated multi- agent systems}

Our multi-robot system is composed of several autonomous agents that can move individually. Then each robot can leave or join a group at any time. Moreover, to limit cost and complexity, we use the same IR emitters/receivers to detect obstacles and to exchange information.

In order to address these problems, we have developed an original dynamic protocol coupling obstacle detection and communication. As many agents must simultaneously emit signals for a long period a classical CSMA/CD-W protocol [13] may be inefficient. Thus, we have adapted a Time Division Multiplexing approach to asynchronous agents.

Each robot's communication is based on a period T. The value of $\mathrm{T}$ is the same for each robot, but the robots are not synchronized. Each can allocate a little part of this cycle to emit a frame. For each cycle, it starts its emission at the same relative time since the beginning of the period. Other agents perceive these emissions at a fixed time in their own cycle because they also use a period of duration $\mathrm{T}$ (see Figure 5). 
When allocating a zone in the cycle each agent chooses a potentially free zone by hearing during a period of $\mathrm{k} \times \mathrm{T}$. If an interference occurs one of the agents makes a new allocation.

Figure 5 shows the dynamic allocation of each agent. On this Figure, agents 1 and 2 are already communicating. When agent 3 arrives into the group, it starts by hearing and analyzing other agents communications to find the free time intervals (hatched areas). During the next period, it takes one of the free places.
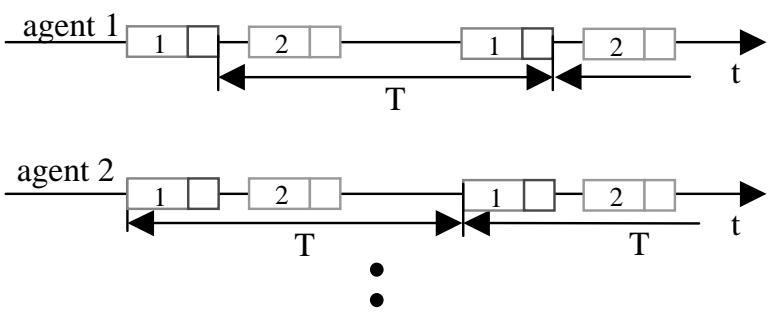

Later agent 3 makes its allocation:

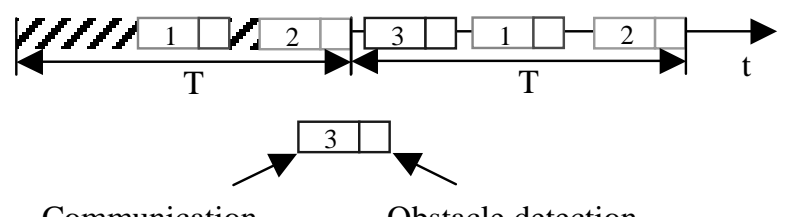

Communication

Obstacle detection

Figure 5: Dynamic allocation

Allocated times are divided into two time periods: the first dedicated to the frame emission and the second to obstacle detection. If during the same period, and on the same sensor, an agent both detects an obstacle and gets a communication message, it can deduce that the obstacle is a robot. Thanks to this method, robots can distinguish communicating agents from environmental obstacles.

In the multi-agent system proposed here each agent communicates only with its $\mathrm{N}$ neighbors. From the radius of communication, this maximal number $\mathrm{N}$ can be computed in order to define the bandwidth $\mathrm{T}=(\mathrm{N}+1)$. Te . Therefore, each agent can always find a time-window in its cycle to communicate.

To implement this protocol and to optimize allocation times, the allocations are shifted on the left whenever possible. As a consequence the size of the free time intervals is increased. Random checks are performed to avoid interferences during transmissions.

\subsection{Applications}

This section addresses the general problem of multirobot navigation in constrained environments.
When many autonomous mobile robots move in the same environment, some conflicts to access to resources may appear. In particular, space may be a source of conflicts (cluttered spaces, corridors, doors, etc.) [10]. Any conflict or deadlock of navigation is insecure both for each agent and for the completion of the common task.

Let us consider a corridor with a dead end (see Figure 6.a) where an agent cannot meet and pass another.

The objective of each robot is to perform a task at the end of the corridor. When the task is completed, the agent must get away. Obviously, a conflicting situation will emerge if the first agent gets out from the corridor while the second one and the others want to enter.

\subsection{Theoretical solution}

Consider the corridor problem with altruistic robots:

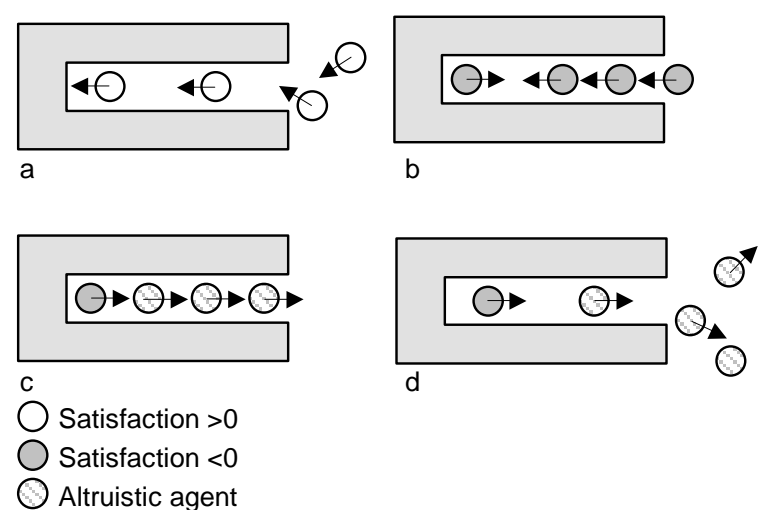

Figure 6: The corridor's problem

1. Figure 6 shows a top view of our problem. At the beginning, all agents are attracted by the goal at the end of the corridor (Figure 6.a) and as a consequence all personal satisfactions are high.

2. After having reached the goal, the first agent tries to go away. The others want to enter into the corridor. The first spatial conflict appears. Then all agent's satisfactions decrease. (Figure 6.b).

3. All obstacles and agents surround the first robot, causing its satisfaction to drop faster than the others.

4. When its satisfaction crosses the threshold of dissatisfaction, a repulsive signal is emitted which repulses its neighbors (Rule 2 ).

5. As the second robot perceives this signal greater than its own dissatisfaction, it switches into the altruistic state. Then it tries to go away and it emits a repulsive signal (Rule 1). 
6. The third robot receives this repulsion and then becomes also altruist. This process continues with the fourth agent and so on (Figure 6.c). Finally, by signal passing, all others agents become altruist and try to go away. Thus, they can go out from the corridor, allowing the first agent to leave the dead end and escape (Figure 6.d).

\section{Results and discussion}

In order to be validated, the presented model has been experimented with the corridor's problem. A closed environment has been build to analyze several conflicts during experiments. After implementing and checking the individual navigator model in a single robot, a multi-robot experiment was realized as follows:

Two robots are initially placed in each side of a closed corridor (Figure 7.a). The aim of each robot is to explore the entire corridor.

During the whole experiment, data (personal satisfaction and current state of the agent) have been stored in each robot. These data were used to draw the graph shown on Figure 8.

\subsection{Description of the experiment}

Quickly the two robots come close to each other (Figure 7.b) and are paralyzed by a conflict: they want to continue their own exploration, i.e. go ahead (Figure 7.c). During this conflict the satisfactions of both robots drop.

The first one to obtain a negative satisfaction emits a repulsive signal, the left robot presented on Figure 8. Immediately the right robot switches into altruistic state and changes its goal to go to the right end of the corridor. (Figure 7.d)

A new problem occurs when the altruistic robot is blocked by the dead end. As it is surrounded by wall obstacles and by the other robot, its satisfaction drops faster that its neighbor's one (Figure 8). Consequently the right robot emits a repulsive signal stronger than the other and repulses the left robot.

The left robot is now the altruistic robot. (Figure 8 and Figures 7.e and 7.f)

As the corridor is not open, the same problem occurs between robots at the left end (Figure 7.g and 8.h). In figure 8 a new solution is shown, because the very dissatisfied left robot succeeds to repulse the other (Figure 7.i and 7.j).

If we open one side of the corridor, the two robots will find quickly the exit, and the problem of conflict is solved.

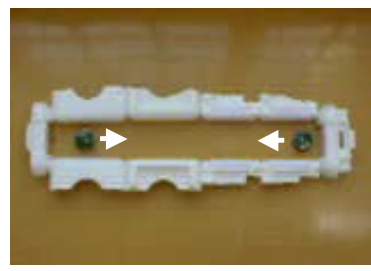

a. start

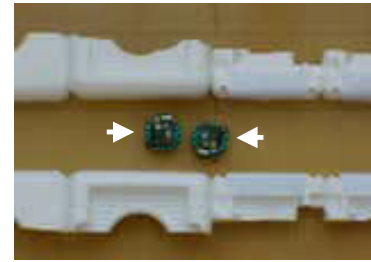

c.

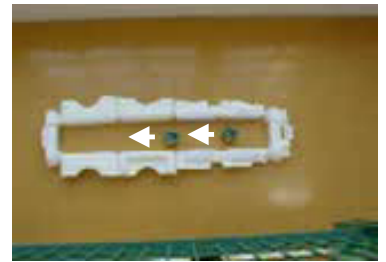

e.

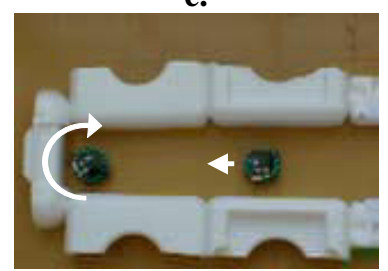

g.

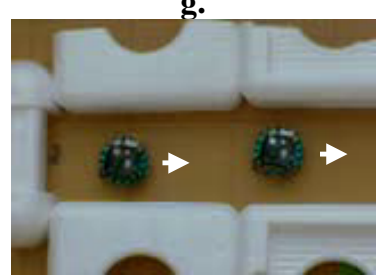

i.

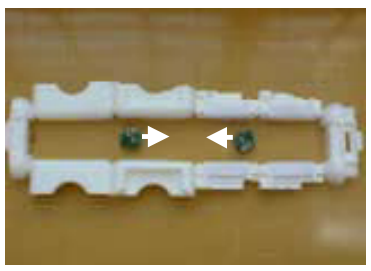

b.

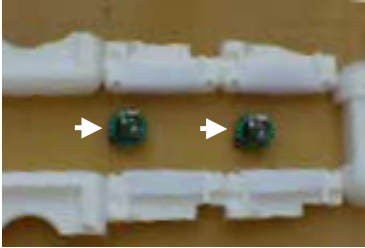

d.

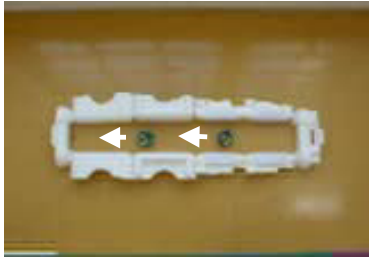

f.

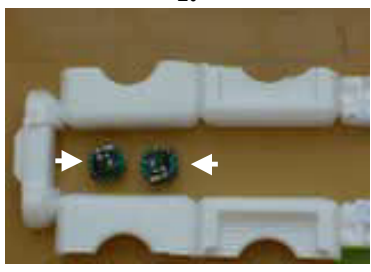

h.

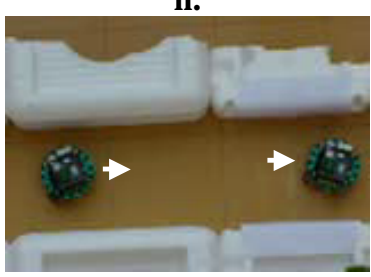

j.
Figure 7: Snapshots of real experiments
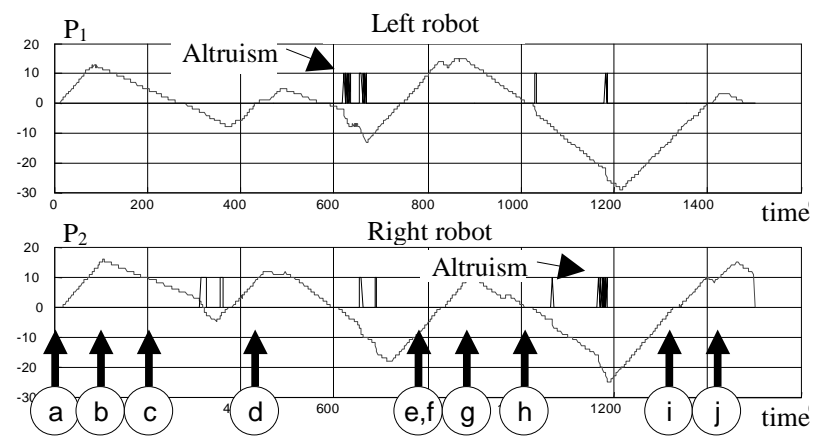

Figure 8: Evolution of personal satisfaction and altruistic state of each robot. 


\subsection{Discussion}

The results of experiments are in a good accordance with the expected ones. First of all, the communication protocol which enables the agents differentiate obstacles from robots works successfully. Robots can also communicate with other agents, even if they have not been synchronized at the beginning. Secondly, the proposed model performs well. The evolution of internal states (as personal satisfaction drawn on Figure 8) shows that the proposed architecture is able to solve spatial conflicts. Simulations with more robots (Figure 9) in more complex environments like in [14] have demonstrated the efficiency of the proposed agent's architecture.
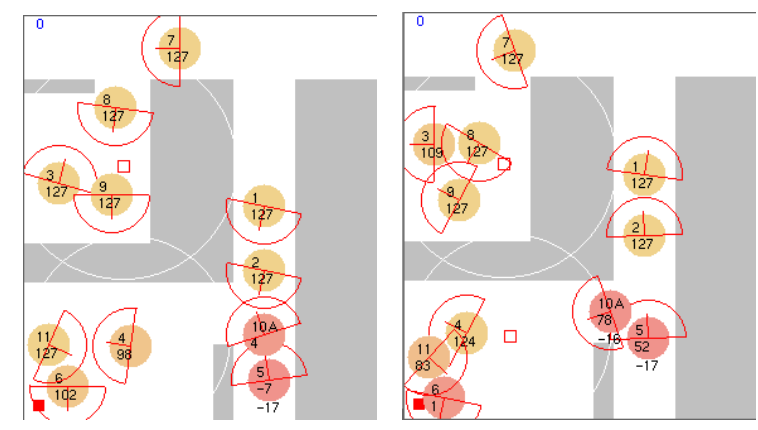

Figure 9: snapshots of a simulation (11 agents in a complex environment).

\section{Conclusion}

In this paper we presented a method for ensuring cooperation between several mobile robots. The coordination and adaptation mechanism, added to the collision avoidance, consists in a state variable called satisfaction emitted by each agent. The experiments showed that the implementation behaves as predicted by the theory and by computer simulations. Experiments are currently performed by using more real robots. An interesting further research direction will be to consider non-homogeneous teams, i.e. made of robots whose sensory, actuation and manipulating capabilities differ. Another direction will consider learning, and adapting to large disturbances like failures.

\section{Acknowledgements}

The STISS Interdisciplinary Program of the University Montpellier 2 has supported parts of the work presented.

\section{References}

[1] S. Botelho and R. Alami, "Multi Robot Cooperation in the Martha Project", IEEE Int. Conf. On Robotics and Automation, pp. 1234-1239, 1999.

[2] T. Kaga, J. Starke, P. Molnar, M. Schanz and T. Fukuda, "Dynamic robot-target assignment dependence of recovering from breakdowns on the speed of the selection process", DARS 4, pp. 325334, 2000.

[3] J. Albus, "Outline for a Theory of Intelligence" IEEE Transactions on Systems, Man and Cybernetics, vol 21, no 3, pp. 473-509, 1991.

[4] M. J. Mataric, "Behavior-Based Control: Examples from Navigation, Learning, and Group Behavior", Journal of Experimental and Theoretical Artificial intelligence, special issue on Software Architectures for Physical Agents, 9(2-3), 1997, 323-336.

[5] O. Simonin, A. Liégeois and P. Rongier, "An Architecture for Reactive Cooperation of Mobile Distributed Robots", DARS 4, pp. 35-44, 2000.

[6] R. Brooks, "A robust layered control system for a mobile robot", IEEE J. of Robotics and Automation 2, pp.14-23, 1986.

[7] T. Balch and R. Arkin, "Motor schema-based formation control for multiagent robot teams" Int. Conf. On Multiagent Systems, pp. 10-16, 1995.

[8] L.E. Parker, "ALLIANCE: An Architecture for Fault Tolerant Multirobot Cooperation", IEEE Trans. on Robotics and Automation, Vol. 14, N ${ }^{\circ} .2$, pp. 220-240, 1998.

[9] O. Khatib, "Real Time Obstacle Avoidance for Manipulators and Mobile robots", IEEE Int. Conf. on Robotics and Automation, pp. 500-505, 1985.

[10] M.S. Fontan and M.J. Mataric, "A Study of Territoriality: The Role of Critical Mass in Adaptive Task Division", In SAB'96, pp. 625-634, 1996.

[11] O. Simonin and J. Ferber, "Modeling Self Satisfaction and Altruism to Handle Action and Reactive Cooperation", in SAB'00 Proceedings Supplement, pp. 314-323, 2000.

[12] P. Lucidarme, P. Rongier and A. Liégeois, "Implementation and Evaluation of a Reactive Multi-Robot System", IEEE Int. Conference on Advanced Intelligent Mechatronics, pp. 165-170, 2001.

[13] J. Wang and S. Premvuti and A. Tabbara, "A Wireless Medium Access Protocol (CSMA/CD-W) for Mobile Robot based Distributed Robotic Systems", IEEE Int. Conf. on Robotics and Automation, pp. 2561-2566, 1995.

[14] R. Vaughan, P. Stoey, G. Sukhatme and M. Mataric "Go ahead, make my days : Robot conflict resolution by aggressive competition" In SAB'00, pp. 11-15, 2000. 